\title{
Matthias Corvinus und Skanderbeg oder die jahrzehntelange Allianz der Häuser Hunyadi und Kastriota im Krieg mit den Osmanen
}

Es gibt wohl keinen geeigneteren Ort als Cluj (Kolozsvár, Klausenburg), um die Beziehungen zwischen den Familien Hunyadi und Kastriota zu behandeln. Denn hier hatte Francisc Pall gewirkt, jener Historiker, der durch die Zeitumstände - den Ausbruch des Zweiten Weltkriegs und danach durch die kommunistische Diktatur in Rumänien - daran gehindert worden war, eine abschließende Monographie zu Skanderbeg zu verfassen, jenem Mann, dem er eine Fülle bedeutender Untersuchungen gewidmet hatte. Was im folgenden vorgestellt werden soll, knüpft an Forschungen Palls an, genauer im Mailänder Staatsarchiv, das Pall im Sommer 1939 besucht hatte, um die mailändischen Gesandtenberichte aus Venedig, Rom und Neapel auszuwerten ${ }^{1}$. Der folgende Vortrag gruppiert sich um einen noch ungedruckten derartigen Gesandtenbericht, der in der Bibliothèque nationale de France aufbewahrt liegt, wo ein Teil des Sforzaarchivs hingelangt ist. Es geht aber nicht nur um die Auswertung eines Texts, sondern vielmehr darum, die Beziehungen zwischen Johann Hunyadi und Matthias Corvinus zu Skanderbeg neu zu interpretieren, auch dies vor dem Hintergrund neuer bzw. neu ausgewerteter Quellen. Es soll die These erhärtet werden, dass die Hunyadi und Skanderbeg von ca. 1440 bis zu Skanderbegs Tod 1468 in einem engen Verhältnis standen, das wesentlich intensiver ausgestaltet war, als bisher angenommen worden ist. Im folgenden gilt der Schwerpunkt den Neuerkenntnissen, Altbekanntes wird hingegen nur gestreift.

1. Dass Hunyadis Vormarsch im Herbst 1443 und Skanderbegs Aufstand zeitlich zusammenfielen, dass Skanderbegs Desertion in der Schlacht von Niš den osmanischen Zusammenbruch und den Sieg des Kreuzfahrerheers auslöste, ist seit langem bekannt. Man hat sich jedoch kaum Gedanken über die Hintergründe dieses Zusammenwirkens gemacht. Dabei handelte es sich um eine konzertierte, von langer Hand geplante Aktion. Denn Skanderbeg und Johann Hunyadi waren sich schon einige Jahre vor der Schlacht bei Niš begegnet. Skanderbeg hatte als osmanischer Offizier in Nikopol an der Donau gedient. Sein erster Biograph, der Bischof von Ulcinj, der aus dem Kosovo stammende und rund zehn Jahre nach Skanderbegs Tod schreibende Martinus Segonus von Novo Brdo, erwähnt ausdrücklich, dass Skanderbeg zusammen mit dem osmanischen Feldherrn Mesid Pascha einen Einfall nach Siebenbürgen unternommen hatte, der von Hunyadi bei Hermannstadt (Nagyszeben, Sibiu) erfolgreich zurückgeschlagen worden war ${ }^{2}$. Ein Bericht des Venezianer Chronisten Stefano Magno schildert zudem eine Flucht Skanderbegs über die Donau, die wohl in Zusammenhang mit der Schlacht an der Ialomiţa im Jahre 1442 zu setzen ist ${ }^{3}$. Weshalb Skanderbeg mit Hunyadi

\footnotetext{
${ }^{1}$ F. PALl, Marino Barlezio: uno storico humanista. Mélanges d'histoire générale 2 (Cluj 1938) 135-318; F. PALL, Die Geschichte Skanderbegs im Lichte der neueren Forschung. Leipziger Vierteljahresschrift für Südosteuropa 6 (1942) 85-98; F. PALL, Le condizioni e gli echi internazionali della lotta antiottomana del 1442-1443, condotta da Giovanni di Hunedoara. RESEE III/3-4 (1965) 433- 463; F. PALL, I rapporti italo-albanesi alla metà del secolo XV. Archivio storico per le provincie napoletane seria III/4 (1965) 123-226; F. PALL, Renseignements inédits sur la participation albanaise à la guerre de Naples (1459-1463), in: Actes du premier congrès international d'études balkaniques et sud-est européennes, Bd. 3 (Histoire). Bukarest 1966, 469-475; F. PALL, Skanderbeg et Ianco de Hunedoara. RESEE VI/1 (1968) 5-21; F. PALL, Di nuovo sulle biografie scanderbegiane del XVI secolo. RESEE IX/1 (1971) 91-106.

${ }^{2}$ Skanderbegs Teilnahme am Angriff auf Siebenbürgen bezeugt Martinus Segonus. Siehe: A. PERTUSI, Martino Segono di Novo Brdo vescovo di Dulcigno. Un umanista serbo-dalmata del tardo Quattrocento (Istituto storico italiano per il medio evo. Studi storici Fasc. 128-130). Rom 1981, 128: "Giorgio Scanderbech, essendo stato rotto nella Transilvania Mesitbech, sotto di cui allhora militava".

${ }^{3}$ Der Chronist Piero Venier in den Annalen des Stefano Magno, in: O. J. SchmiTt, Die Venezianischen Jahrbücher des Stefano Magno als Quelle für die albanische und epirotische Geschichte (1433-1477), in: Südosteuropa. Von vormoderner Vielfalt und nationalstaatlicher Vereinheitlichung, ed. K. Clewing - O. J. SchmitT. München 2005, 133-182, hier 137; M. CAZACU, Dracula. Paris 2004, 71f.; E. C. ANTOCHE, La bataille de la rivière de Ialomiţa (2 septembre 1442), une victoire majeure de la Chrétienté
} 
zusammenarbeitete, geht wiederum aus einem unedierten Mailänder Dispaccio aus dem Jahr 1454 hervor: Skanderbegs Motiv war Blutrache, da Murad II. Skanderbegs Vater Ivan Kastriota hatte töten lassen ${ }^{4}$. Nur deshalb kehrte der erfolgreiche Renegat zum Christentum zurück: er war durch das Gewohnheitsrecht gezwungen, Rache zu nehmen. Skanderbegs Vater starb im Mai 1437; danach wurde Skanderbeg an die Donau versetzt, wo er auf eine Gelegenheit zur Blutrache wartete ${ }^{5}$. Die Rache gelang im Sommer 1443: die Mailänder Quelle erzählt, dass Skanderbeg den Bruder Mehmeds II. habe ermorden lassen. Dabei handelte es sich um den Kronprinzen Alaeddin Ali Celebi, der in Anatolien umgebracht worden war. Indizien legen nahe, dass in den Anschlag wichtige Personen am osmanischen Hof verwickelt waren, unter anderem Mara Branković und Prinz Mehmed selbst. Skanderbeg war mütterlicherseits wohl ein Brankovićc; Mara hatte ebenfalls ein starkes Motiv - Rache für ihre geblendeten Brüder ${ }^{7}$. Die Verschwörer wollten aber das osmanische Reich ganz vernichten, und dafür benötigten sie Hilfe von außen, in Anatolien durch den Emir von Karaman, in Südosteuropa durch einen Kreuzzug. Diesen werden Skanderbeg und Hunyadi genau vorbereitet haben: die zeitliche Abstimmung von Angriff und Desertion sprechen ebenso dafür wie die spätere enge Zusammenarbeit Skanderbegs und Hunyadis, besonders im Jahre 1448. Als wesentliches Hindernis erwies sich 1444 wie 1448 die Haltung der Brankovići, d. h. des serbischen Despotats: während Hunyadi und Skanderbeg aus unterschiedlichen Motiven die Vernichtung der Osmanen wünschten, fürchteten die orthodoxen Serben ein Übergewicht des katholischen Ungarn auf dem Balkan - die Politik der Äquidistanz zwischen Ungarn und den Osmanen scheiterte aber gänzlich; einer der letzten Branković floh zu Skanderbeg, dem alten Verbündeten; Skanderbegs Sohn Ivan heiratete in der Folge eine Prinzessin aus dem Hause Branković ${ }^{8}$. Auch dies belegt, wie tief die Dreiecksbeziehung Kastriota-Branković-Hunyadi war.

2. Nach dem Zusammenbruch des Feldzugs Hunyadis 1448 war die Achse Skanderbeg-Ungarn geschwächt. Dies bedeutete aber nicht, dass die Hunyadi die Option eines Balkanbündnisses, d. h. eines Zangenangriffs aufgegeben hätten. Im Gegenteil, der junge Matthias Corvinus setzte die Politik seines Vaters unmittelbar nach seinem Thronantritt fort. Der junge ungarische König Matthias Corvinus war im Herbst 1458 erstmals in die Fussstapfen seines Vaters Johann Hunyadi getreten und hatte die Osmanen an seiner Reichsgrenze geschlagen ${ }^{9}$. Papst Kalixt III. hatte ihn bei seiner Thronbesteigung in einem Schreiben am 14. März 1458 ermahnt, das Werk Hunyadis fortzusetzen und dabei ausdrücklich auf „unseren lieben Sohn, den vornehmen Herrn Skanderbeg in Albanien“ hingewiesen ${ }^{10}$. Matthias ging sogleich auf diese Bitte ein; wieder diente das nominell ungarische Dubrovnik (Ragusa) als Drehscheibe. Im Februar 1459 reiste Corvinus' Gesandter, der Serbe Stupko aus Belgrad, zu Skanderbeg; der Inhalt der Verhandlungen ist nicht bekannt; der Ragusaner Senat schrieb am 24. Februar seinem Oberherrn lediglich, dass der Diplomat auf einem Schiff der

face aux armées ottomanes. Cahiers du Centre d'études d'histoire de la défense 9 (1999) 61 -88; E. C. AnTochE, Le rayonnement de l'art militaire hussite dans l'Europe orientale et le Moyen Orient (XVe-XVIIe siècles). Revista istorică 14/5-6 (2003) 87-109, hier 97ff.

${ }^{4}$ Archivio di Stato di Milano. Archivio visconteo - sforzesco B. 41 Bericht der Diplomaten Sceva de Curte und Jacobo Trivulzio an Herzog Francesco Sforza. Rom 10. Jänner 1454: "Qua è gionto uno cavalero ambasatore d'uno Signore chi dice de Grecia e chi de Albania chiamato quel Signor Xandrebech et è infidele ... per hodio privato ha cum el Turcho, lo quale Turcho fece morire lo padre de quel tal Signor e questo tal Signore ha facto morire uno fratello del Turcho".

${ }^{5}$ N. Todorov - B. NedKov, Turski izvori za bălgarskata istorija. Serija XV-XVI/2. Sofia 1966, 161. Das Verzeichnis wird von den Herausgebern in die Mitte des 15. Jahrhunderts datiert. Siehe zu Nikopol: R. P. KovaČEv, Opis na Nikopolskija sandžak ot 80-te godini na XV vek. Sofia 1997; E. RADOUŠEv, Ottoman Border Periphery (serhad) in the Nikopol Vilayet, First Half of the 16th Century. Études balkaniques 31/3-4 (1995) 140-160. Eine eingehende Diskussion der Frage auch bei L. MALLTEZI, Një dokument i ri për Skënderbeun. eksklusive (in Prishtinë/Priština veröffentlichtes Magazin; Anm. des Verfassers) 20 (2001) 61-64; das von ihm veröffentlichte Stück bietet aber keinen Beweis für einen Aufenthalt Skanderbegs in Albanien nach 1438.

${ }^{6}$ B. Petrovski, Voisava Tribalda, in: Gjergj Kastrioti Skenderbeg 1405-1468, ed. Institut za Nacionalna Istorija. Skopje 2006, $67-78$.

${ }^{7}$ Zu ihr: M. St. Popović, Mara Branković. Eine Frau zwischen dem christlichen und dem islamischen Kulturkreis im 15. Jahrhundert (Peleus, Studien zur Archäologie und Geschichte Griechenlands und Zyperns 45). Mainz - Ruhpolding 2010.

${ }^{8}$ Popović, Mara Branković, 135f.; M. SPREMIĆ, Despot Đurađ Branković i njegovo doba. Belgrad 1994, 646.

${ }^{9}$ G. RÁszó, Die Türkenpolitik Matthias' Corvinus. Acta Historica Academiae Scientiarum Hungaricae 32 (1986) 3-50; S. GRACIOTTI (Ed.), Italia e Ungheria all'epoca dell'umanesimo corviniano. Florenz 1994 sowie S. GRACIOTTI (Ed.), Spiritualità e lettere nella cultura italiana e ungherese del basso medioevo. Florenz 1995.

10 I. PARrino, Acta Albaniae Vaticana Bd. 1. Vatikan 1971, Nr. 314. 
Ragusaner nach Albanien in See gestochen sei $^{11}$. Ein knappes halbes Jahr später unternahm der König eine energische Demarche in Venedig zugunsten Skanderbegs, der sich offensichtlich über die Feindseligkeit der Signoria beklagt hatte. Weiter verlangte der König, die Republik möge den Kastriota in einen Frieden mit dem Sultan einbeziehen. Der Senat versprach zwar keine Hilfe im Türkenkrieg, zeigte sich aber bereit, allfällige Streitigkeit durch Verhandlungen zu lösen, wie, so erinnerten die Venezianer den ungarischen Gesandten, dies ja eben geschehen $\mathrm{se}^{12}$. Skanderbeg hatte in Matthias Corvinus einen neuen, wenn auch fernen Schutzherrn gefunden, der für ihn in Italien ein gutes Wort einlegte. Matthias seinerseits strebte danach, seine Südgrenze zu schützen; eine Stärkung Skanderbegs kam ihm dabei gelegen. Eigentliche Truppenhilfe vermochte er nicht zu leisten, zumal er nicht einmal zu verhindern imstande war, dass die Osmanen am 20. Juni 1459 ohne Schwertstreich Smederevo einnahmen und damit das serbische Despotat endgültig beseitig$\operatorname{ten}^{13}$. Die Sultanstruppen standen erneut bedrohlich vor Belgrad. In der Folge kam Corvinus in päpstlichen Planspielen, besonders Pius' II., stets eine besondere Rolle zu als Herrscher einer kontinentalen Flankenmacht bei einem Zangenangriff auf das osmanische Reich. Als es 1464 jedoch ernst wurde, meinte Pius II., König Matthias werde sich kaum den Weg nach Dalmatien freikämpfen ${ }^{14}$. Auch Venedig bezog Ungarn in sein Kalkül ein und schloss mit Corvinus den bekannten Vertrag von September 1463. Für keinen anderen aber war Corvinus wichtiger als für Skanderbeg: dessen venezianisch-albanischer Angriffskrieg war im Herbst 1464 in einem völligen Fehlschlag geendet. Das alte Misstrauen auf beiden Seiten erwachte wieder; denn Skanderbeg hatte sich im August 1464 an Dubrovnik gewandt und vorgeschlagen, selbst in die Blasiusstadt zu kommen ${ }^{15}$. Dies kann kaum anders denn als Rückversicherung verstanden werden, zudem auch als Vorbereitung von Verhandlungen mit dem ungarischen König Matthias Corvinus, der in Bosnien gegen das osmanische Heer kämpfte. Schon nach wenigen Monaten sah Skanderbeg das Bündnis mit Venedig als gescheitert an. Der Sohn seines alten Waffengefährten Johann Hunyadi erschien ihm ein weit stärkerer Bundesgenosse, der in der Lage war, mit seinem Landheer tief nach Bosnien vorzustossen. Im Gegensatz zu den Venezianern erzielten die Ungarn in den Bergen des Balkans Erfolge. Im Dezember 1463 hatten sie die Osmanen aus der alten bosnischen Königsburg Jajce vertrieben und sich, was noch viel grösseren Eindruck hervorrief, im August 1464 gegen einen Angriff, den Mehmed II. persönlich anführte, behauptet ${ }^{16}$. Ein Vorstoß gegen Zvornik aber scheiterte, und König Matthias musste weite Teile des gewonnenen Gebietes wieder räumen. Damit zerschlugen sich hochfliegende Pläne: denn im Oktober 1463 hatten die Ragusaner Skanderbeg den Einmarsch des Königs in Bosnien gemeldet, und Anfang September 1464 hatte Matthias Corvinus aus seinem Feldlager zu Gara in Slawonien Skanderbeg geschrieben und eine Wiederholung jenes Zangenangriffes vorgeschlagen, den 16 Jahre zuvor schon sein Vater Johann Hunyadi geplant hatte ${ }^{17}$. Sowohl Venedig als auch Mehmed II. reagierten auf die Entwicklungen in Albanien. Venedig beauftragte Giovanni Emo, seinen Gesandten beim ungarischen König Matthias Corvinus, auf einen ungarischen Vorstoß nach Süden und eine Vereinigung der ungarischen Truppen mit Skanderbeg zu drängen ${ }^{18}$. Damit sollten auch die venezianischen Truppen in der Morea entlastet werden, die nach schnellen Anfangserfolgen schon bald in ernste

11 J. RADONIĆ, Đurađ Kastriot Skenderbeg i Albanija u XV veku. Belgrad 1942, Nr. 175, 178.

12 RAdOniĆ, Đurađ Kastriot Skenderbeg, Nr. 183; J. VALEnTINI, Acta Albaniae Veneta saeculorum XIV et XV. 25 Bde. München Palermo - Mailand 1967-1975, Nr. 6798.

${ }^{13}$ F. BABINGER, Mehmed der Eroberer. Weltenstürmer einer Zeitenwende. München - Zürich 1987, 174; SPREMIĆ, Despot Đurađ Branković, 637.

${ }^{14}$ O. J. Sснміт, Skanderbeg als neuer Alexander: Antikerezeption im spätmittelalterlichen Albanien. Pirckheimer-Jahrbuch 20 (2005) 123-144.

15 RADONIĆ, Đurađ Kastriot Skenderbeg, Nr. 276, 277 (Auszug aus der Chronik des Ragusaners Junius Resti).

${ }^{16}$ BABINGER, Mehmed, 245ff.; J. MrgiĆ-RAdOJČIĆ, Donji Kraji. Krajina srednjovekovne Bosne. Belgrad 2002, $125 \mathrm{ff}$.

17 Ć. Truhelka, Dubrovačke vijesti o godini 1463. Glasnik Zemaljskog Muzeja u Bosni i Hercegovini 22 (1910) 1-24, hier 23; Bibliothèque nationale de France (= BNF). Manuscrits italiens 1590 f. 381v. Kopie eines Schreibens des venezianischen Gesandten im ungarischen Heerlager von Gara, 6. September 1464 ("zà ha scripto Sua Maestà a Schanderbech che quella farà se possa unir cum lui. Diseme etiam ch'io scrivesse a Vostra Signoria che comandasse a quelle zente de Albania che acorendo bixogno se potessemo unirse cum Sua Maestà"); vgl. auch den Bericht des spätbyzantinischen Geschichtsschreibers Kritobulos (bei RADONIĆ, Đurađ Kastriot Skenderbeg, 226). Der Plan war den Osmanen bekannt, sonst hätte ihn der Geschichtsschreiber aus Imbros nicht erzählen können. Ein Zangenangriff wurde auch noch im Dezember in Rom diskutiert (BNF. Manuscrits italiens $1590 \mathrm{f}$. 513).

18 RADONIĆ, Đurađ Kastriot Skenderbeg, Nr. 284. 
Bedrängnis geraten waren. Skanderbeg blieb gewiss nicht verborgen, dass Venedigs Unterstützung mehr als nur lau war; da er selbst sein Abkommen mit Mehmed II. in der Hoffnung auf den Kreuzzug gebrochen hatte und er zudem trotz seiner Tätigkeit als Vermittler von den Osmanen keine Garantien für sein mittelalbanisches Machtgebiet erhalten hatte, bemühte er sich um eine Absicherung seiner gefährdeten Lage. Im Dezember 1464 führte er mit Helena Branković Verhandlungen über die Heirat seines neunjährigen Sohnes Ivan mit der achtjährigen Jerina ${ }^{19}$. Besonders enge Beziehungen unterhielt er zu Dubrovnik, das ihm im Februar 1465 Bauleute zur Fertigstellung seiner Fluchtburg bei Kap Rodoni gewährte, und zu König Matthias Corvinus von Ungarn ${ }^{20}$. Dieser schickte im Hochsommer 1465 den Frater Alexander als Botschafter zu Skanderbeg $^{21}$; Dubrovnik unterstützte diese Gesandtschaft und beauftragte am 12. August 1465 den Patrizier Paladino de Luccari/Lukarević, den Mönch auf einem ragusanischen Schiff nach Albanien zu geleiten ${ }^{22}$. Am 15. Dezember desselben Jahres befand sich besagter Frater wieder in Ragusa. Über den Inhalt seiner Gesandtschaft ist nichts bekannt. Aus den ragusanischen Ratsbüchern geht aber hervor, dass die Blasiusrepublik der Bitte Skanderbegs stattgab, den Patrizier Paladino de Gondola/Gundulić als Gesandten des Albanerfürsten zu Matthias Corvinus zu schicken (13. November 1465) ${ }^{23}$. Man darf annehmen, dass es bei diesen Gesandtschaften auch um die Friedensfühler ging, die Mehmed II. zum ungarischen König ausstreckte, und dass Matthias im Andenken an die Waffenbrüderschaft seines Vaters Johann Hunyadi mit Skanderbeg diesen in den geplanten Waffenstillstand miteinschliessen wollte. Die christlichen Verbündeten hatten sich freilich darauf festgelegt, auf einen Separatfrieden mit dem Sultan zu verzichten. Die Verhandlungen mit Matthias Corvinus hatten Mehmed II. jedoch nicht daran gehindert, gegen ungarische Interessen in Bosnien vorzugehen und so den Druck auf den König zu erhöhen, für den der Kampf gegen die Osmanen nie zu den vorrangigen Zielen seiner Herrschaft zählte ${ }^{24}$.

Als ihn Mehmed II. mit einem Grossangriff an den Rand des Untergangs brachte, wandte sich Skanderbeg 1466 wieder an den Sohn seines alten Verbündeten Johann Hunyadi. Seit dem Beginn des Krieges hatte zwischen König Matthias Corvinus und Skanderbeg ein reger Gesandtenverkehr geherrscht; nach dem gescheiterten Vorstoß in Bosnien 1464 hatte sich der ungarische Monarch aber zurückgehalten. Im Herbst 1466 machte sich im Auftrag Skanderbegs der Ragusaner Paladino de Gondola/Gundulić auf den Weg nach Nor$\operatorname{den}^{25}$. Der Rat seiner Republik beschied ihm, er habe aus Dubrovnik selbst nichts auszurichten. Ragusa hatte Skanderbeg verloren gegeben, unterstützte jedoch im Geheimen private Hilfsaktionen Ragusaner Patrizier. Auch dem Knezen Stjepan Radojević, einem weiteren Gesandten des Kastriota, erlaubte man die Reise nach Šibenik oder Split, von wo aus er sich wohl zu Corvinus begeben wollte ${ }^{26}$. Zu erwarten war von Ungarn nicht viel. Dies zeigte sich im Folgejahr, als Mehmed II. Skanderbeg endgültig besiegte: Corvinus ging auf das osmanische Anerbieten ein und schickte einen Botschafter nach Sofia, wo der Sultan Truppen für seinen Albanienfeldzug sammelte ${ }^{27}$. So hielt der Großherr sich den Rücken frei. Seine Widersacher im Abendland waren zerstritten oder durch geschickte Diplomatie ausgespielt. Der Weg nach Albanien stand offen.

\footnotetext{
19 BNF. Manuscrits italiens 1590 f. 523.

${ }^{20}$ RADONIĆ, Đurađ Kastriot Skenderbeg, Nr. 167; L. NADIN, Capo Rodoni nella storia, in: L. ZÀ (unter Mitarbeit von L. NADIN), I villaggi del Dio Rodon. Frammenti di vita rurale albanese. Lecce 2001, 25f., $31 \mathrm{f}$.

${ }^{21}$ RADONIĆ, Đurađ Kastriot Skenderbeg, Nr. 274, 299.

${ }^{22}$ IDEM, Nr. 308.

${ }^{23}$ IDEM, Nr. 306.

${ }^{24}$ Vgl. K. Nehring, Matthias Corvinus, Kaiser Friedrich III. und das Reich. München 1989; J. K. HoEnSCH, Matthias Corvinus. Diplomat, Feldherr und Mäzen. Graz 1998.

${ }^{25}$ Državni arhiv u Dubrovniku. Consilium rogatorum 3/19 f. 136v, 138 r.

${ }^{26}$ Državni arhiv u Dubrovniku. Diversa Notariae 26/49 f. 142v-143r.

27 O. J. Schmitт, Skanderbegs letzte Jahre - West-östliches Wechselspiel von Diplomatie und Krieg (1464-1468). SüdostForschungen 63/64 (2004/2005) 56-123, Nr. 40, 45. Venedig und Ungarn benachrichtigten sich wechselseitig über ihre jeweiligen Verhandlungen mit dem Sultan, wobei die Furcht mitspielte, der andere Bundesgenosse könnte einen Separatfrieden schliessen; I. NAGY - A. NYÁRY, Magyar diplomacziai emlékek Mátyás király korából 1458-1490. 4 Bde. Budapest 1875-1878, Bd. 2, 50-52, 56-58, 64-66. Am 6. August 1467 traf in Venedig eine nicht weniger als 756 Reiter zählende ungarische Gesandtschaft ein (Ebd. 66f.).
} 
Zusammenfassend lässt sich feststellen, dass die Skanderbeg und die beiden Hunyadi insgesamt dreimal einen Zangenangriff auf die Osmanen unternahmen - 1443, 1448 und 1464 - dass man also von einer zwei Jahrzehnte dauernden Tradition regionaler Angriffspläne gegen die Osmanen sprechen kann. Nach dem Scheitern beider Verbündeter, Skanderbegs im heutigen Westmakedonien, Corvinus' in Bosnien, vor allem aber nach den verheerenden Rachefeldzügen Mehmeds II. gegen Albanien, besaß das Bündnis keine militärische Grundlage mehr. Für das Königreich Ungarn wie für das Aufstandsgebiet Skanderbegs im westlichen Balkan zeitigte das Scheitern der Allianz langfristig die bekannten schweren Folgen. 
